\title{
Abelian Primitive Words
}

\author{
Michael Domaratzki \\ Department of Computer Science \\ University of Manitoba \\ Winnipeg, MB R3T 2N2 \\ Canada \\ mdomaratecs.umanitoba.ca
}

\author{
Narad Rampersad \\ Department of Mathematics \\ University of Liège \\ 4000 Liège \\ Belgium \\ narad.rampersadegmail.com
}

\begin{abstract}
We investigate Abelian primitive words, which are words that are not Abelian powers. We show the set of Abelian primitive words is not context-free. We can determine whether a word is Abelian primitive in linear time. Also different from classical primitive words, we find that a word may have more than one Abelian root. We also consider enumeration of Abelian primitive words.
\end{abstract}

\section{Introduction}

Repetition in words is a well-studied topic, and many of the results in this area can be classified into two distinct research areas: the theory of formal languages and the study of combinatorics on words. In these two areas, the focus on repetition is slightly different: in formal language theory, research focuses on the properties of languages containing words with different types of repetition, while in combinatorics on words, research typically concentrates on the existence or non-existence of individual words which avoid certain repetitions, and combinatorial enumeration of words with or without repetitions.

An example of a long-standing area of research relating to repetition in both the theory of formal languages and combinatorics on words are primitive words: a word $x$ is primitive if it cannot be expressed as a repetition of some shorter word $y$. In combinatorics on words, an elegant proof of the number of primitive words of length is given using Möbius inversions (see, e.g., Lothaire [14]). However, in formal language theory, it is unknown whether the set of primitive words are a context-free language or not (see, e.g., Dömösi et al. [10]). However, it is known that a closely related set, the set of Lyndon words, is not context-free [4].

In combinatorics on words, a parallel notion to standard repetition is Abelian repetition. A word $x$ is an Abelian power if it can be divided into blocks $x=x_{1} x_{2} \cdots x_{n}$ where every block $x_{i}$ is a permutation of every other block.

In this paper, we consider the application of Abelian repetition to the concept of primitivity. Despite the naturalness of this application, the concept does not appear to have attracted much attention before 1 . In a related concept, Czeizler et al. [7] study repetitions with only limited rearrangement. We study the language of Abelian primitive words, a formal language theoretic question, as well as the number of Abelian primitive words of a given length, a problem in combinatorics on words.

\section{Definitions}

For additional background in formal languages and automata theory, see Rozenberg and A. Salomaa [17]. Let $\Sigma$ be a finite set of letters, called an alphabet. A string over $\Sigma$ is any finite sequence of letters from $\Sigma$. The string containing no symbols, the empty string, is denoted $\varepsilon$. The set $\Sigma^{*}$ is the set of all strings over $\Sigma$. A language $L$ is any subset of

\footnotetext{
${ }^{1}$ We have found a reference to a research project studying Abelian primitive words on the web at http://bit. ly/9NWqSI but have been unable to obtain a copy of any associated works.
} 
$\Sigma^{*}$. If $x=a_{1} a_{2} \cdots a_{n}$ is a string, with $a_{i} \in \Sigma$, then the length of $x$, denoted by $|x|$, is $n$. For $a \in \Sigma$ and $w \in \Sigma,|w|_{a}$ is the number of occurrences of $a$ in $w$.

For languages $L_{1}, L_{2} \subseteq \Sigma^{*}$ the left quotient of $L_{1}$ by $L_{2}$, denoted $L_{2} \backslash L_{1}$, is defined by

$$
L_{2} \backslash L_{1}=\left\{x \in \Sigma^{*}: \exists y \in L_{2} \text { such that } y x \in L_{1}\right\} .
$$

Given an (ordered) alphabet $\Sigma=\left\{a_{1}, \ldots, a_{n}\right\}$, the Parikh vector of a word $w \in \Sigma^{*}$ is $\Psi(w)=\left(|w| a_{1},|w| a_{2}, \ldots,|w| a_{n}\right)$. For the alphabet $\Sigma=\{a, b\}$, we assume $a<b$. Thus, for example $\Psi(a b b a b)=(2,3)$.

We first recall the standard notions of primitive words. A word $w$ is primitive if $w$ cannot be written as $z^{k}$ for $z \in \Sigma^{*}$ and $k \geq 2$. If $w$ is not primitive, then there is a unique primitive word $u$ such that $w=u^{k}$ for some $k \geq 2$. For an alphabet $\Sigma$, the set of all primitive words $w \in \Sigma^{*}$ is denoted $Q(\Sigma)$ or simply $Q$ if $\Sigma$ is understood.

We now turn to the generalization of these notions to Abelian repetitions. A word $w$ is a $n$-th Abelian power if $w=u_{1} u_{2} \cdots u_{n}$ for some $u_{1}, u_{2}, \ldots, u_{n}$ such that for all $1 \leq i, j \leq n, \Psi\left(u_{i}\right)=\Psi\left(u_{j}\right)$. That is, each $u_{j}$ with $j \geq 2$ is a permutation of $u_{1}$.

We say that a word $w$ is Abelian primitive (or A-primitive, for short) if $w$ fails to be a $k$-th Abelian power for every $k \geq 2$. For an alphabet $\Sigma$, the set of all A-primitive words $w \in \Sigma^{*}$ is denoted by $A Q(\Sigma)$ or simply $A Q$ if $\Sigma$ is understood.

Example 1. The word $w=a a b b a b$ is A-primitive, while $u=a a b b a b a b$ is not, as $u=x y$ where $\Psi(x)=\Psi(y)=(2,2)$.

Let $w$ be an Abelian power. Then we say that an word $u$ is an Abelian root (or A-root) of $w$ if $w=u u_{1} u_{2} \cdots u_{n}$ for some $u_{1}, \ldots, u_{n} \in \Sigma^{*}$ with $\Psi(u)=\Psi\left(u_{i}\right)$ for all $1 \leq i \leq n$. If $w$ has an A-root $u$ which is also A-primitive, then we say that $u$ is an A-primitive root of $w$. Two A-primitive roots $u, v$ of a word $w$ are distinct if $|u|$ does not divide $|v|$ or vice versa. On the other hand, we note the following simple but useful fact:

Observation 1. If a word $x$ has an A-root of length $k$, then $x$ also has an A-root of length $k^{\prime}$ for all $k^{\prime}$ where $k$ divides $k^{\prime}$ and $k^{\prime}$ divides $n$.

We recall some notation from number theory. Recall that if $r, z$ are integers, $r \mid z$ denotes that $r$ divides $z$, i.e., $z=r k$ for some $k \geq 0$. We say that a set of integers $S$ is division-free if $x \nmid y$ and $y \nmid x$ for all $x, y \in S$. For all $n \geq 2$, let $\omega(n)$ denote the number of prime divisors of $n$, while $\omega^{\prime}(n)$ is the number of prime divisors of $n$ with multiplicity 2 . Thus, if $n \geq 2$ and $n=p_{1}^{\alpha_{1}} p_{2}^{\alpha_{2}} \cdots p_{k}^{\alpha_{k}}$ is its prime factorization, then $\omega(n)=k$ and $\omega^{\prime}(n)=\sum_{i=1}^{k} \alpha_{i}$. We also let $d(n)$ be the number of divisors of $n$, i.e., $d(n)=\prod_{i=1}^{k}\left(1+\alpha_{i}\right)$.

\section{Non-context-freeness of $A Q$}

We now show that the set $A Q$ of all A-primitive words is not context-free. This is in contrast to the set of ordinary primitive words $Q$, for which it is unknown whether they are a context-free language or not. We begin with two preliminary propositions.

Proposition 1. Let $p$ be a prime and $x=a a b b(a b)^{p-2}$. Then $x$ is A-primitive.

Proof. Note that $|x|=2 p$. If $x$ is not A-primitive, then one of three cases occurs:

(a) $x=u^{2 p}$ for some letter $u$,

(b) $x=u_{1} u_{2} \cdots u_{p}$ for words $u_{1}, \ldots, u_{p}$ of length two, or

(c) $x=v_{1} v_{2}$ for words $v_{1}, v_{2}$ of length $p$.

The first of these possibilities cannot occur, as $x$ contains occurrences of both $a$ and $b$. The second case is also not possible, since if so, we would have $u_{1}=a a$ and $u_{2}=b b$, which do not have matching Parikh vectors. Thus, we must have that $x=v_{1} v_{2}$ for $\left|v_{1}\right|=\left|v_{2}\right|=p$. We have three subcases:

\footnotetext{
${ }^{2}$ The notation $\Omega(n)$ is also used for what we call $\omega^{\prime}(n)$, but we reserve $\Omega$ for denoting asymptotic function growth. Our notation is from Bach and Shallit [3].
} 
(a) if $p=2$, then we are in the previous case, i.e., $v_{1}=a a$.

(b) if $p=3$, then $v_{1}=a a b$ and $v_{2}=b a b$.

(c) otherwise $p>3$ and $v_{1}=a a b b(a b)^{(p-5) / 2} a$ which has Parikh vector $((p-5) / 2+3,(p-5) / 2+2)$, and $v_{2}=$ $b(a b)^{(p-1) / 2}$ which has Parikh vector $((p-1) / 2,(p-1) / 2+1)$. We can see that the number of occurrences of $a$ in $v_{1}$ is even, while in $v_{2}$ it is odd or vice versa.

Proposition 2. Let $M=A Q \cap a a b b(a b)^{*}$. Then

$$
M=\left\{a a b b(a b)^{p-2}: p \text { is prime. }\right\} .
$$

Proof. The right-to-left inclusion is immediate from Proposition 1

For the reverse inclusion, let $x \in M$. Then $|x|=2 n$ for some $n \geq 2$. Suppose, contrary to what we want to prove, that $x$ is not of the form $a a b b(a b)^{p-2}$ for some prime $p$. Then we must have that $n$ is not prime. Let $q$ be a prime factor of $n$ and note that

$$
x=\left(a a b b(a b)^{q-2}\right) \cdot\left((a b)^{q}\right)^{n / q-1}
$$

and that all factors of length $2 q$ have $q$ occurrences of $a$ and $q$ occurrences of $b$. Further, $a a b b(a b)^{q-2}$ is an A-primitive root by Proposition 1 .

We can now show that the set of all $A$-primitive words is not context-free.

Theorem 1. The set $A Q$ is not context-free.

Proof. We prove that $M$ is not context-free. Let $M^{\prime}=h^{-1}(\{a a b b\} \backslash M)$ where $h:\{a\}^{*} \rightarrow\{a, b\}^{*}$ is the morphism $h(a)=a b$. Then $M^{\prime}=\left\{a^{p-2}: p\right.$ is prime $\}$. As the context-free languages are closed under quotient by regular sets and inverse homomorphism, $M^{\prime}$ is context-free if $M$ is. But as $M^{\prime}$ is unary, if it is a context-free language then it is also regular. But by the pumping lemma, we can see that $M^{\prime}$ is not regular. Thus, neither are $M$ or $A Q$.

The set of all non-trivial Abelian powers, $\overline{A Q}$, is also non-context-free, as can be seen through, e.g., the intersection

$$
\overline{A Q} \cap a^{*} b a^{*} b a^{*} b=\left\{a^{n} b a^{n} b a^{n} b: n \geq 0\right\} .
$$

For discussion on the complexity of the language of marked Abelian squares and its relation to iterated shuffle and deletion operations, see Domaratzki [9, Sect. 8.4.1] and Jędrezejowicz and Szepietowski [13, Ex. 3.2]. Using the interchange lemma, Gabarró [11] has proven that the language $\left\{u w_{1} w_{2} v: u, w_{1}, w_{2}, v \in \Sigma^{*}, \Psi\left(w_{1}\right)=\Psi\left(w_{2}\right)\right\}$ of words containing an Abelian square is not context-free.

\section{Complexity of $A Q$}

Through an elegant pattern matching algorithm [15, Thm. 13], it is known that we can determine whether a word is primitive in linear time. We now consider this problem for A-primitive words. Throughout this section, we consider the size of the alphabet to be a fixed constant. In order to illustrate the basic principles of the algorithm, we begin with an $O(n \log n / \log \log n)$ algorithm:

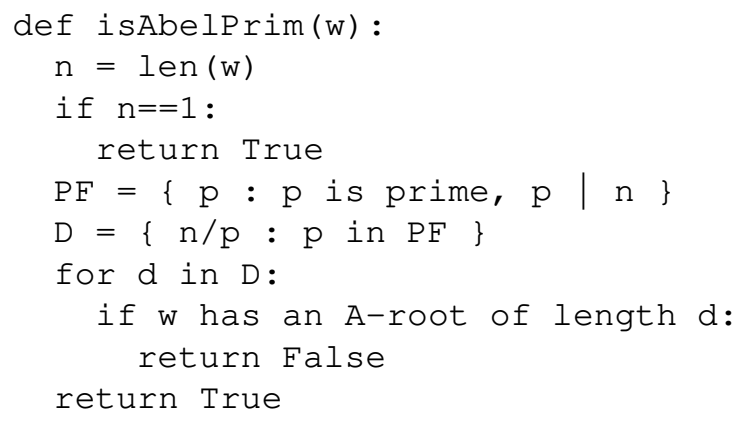


Suppose that $w \in A Q$. Then $w$ certainly does not have a A-root whose length is any the periods in $D$, thus is AbelPrim returns true. On the other hand, if $w \notin A Q$ with $|w|>1$, then $w$ has an A-root of length $r$ for some $r \mid n$ with $r<n$. There exists $d_{r} \in D$ such that $r \mid d_{r}$ ( $r$ may also divide other $d \in D$, but it is enough to know it divides some $d_{r}$ ). By Observation 1, on the loop of is

One iteration of the loop in isAbelPrim will take time $O(n)$, by walking across $w$ and computing the Parikh vectors for each block of length $d \in D$. Thus, the runtime of the algorithm is $O(p(n)+n \omega(n))$ where $p(n)$ is the time required to calculate the set $\mathrm{PF}$.

We claim that even using trial division (rather than more complex methods such as, e.g., general number field sieve [6]), we have $p(n) \in O(\sqrt{n} \log n)$. Consider the following algorithm:

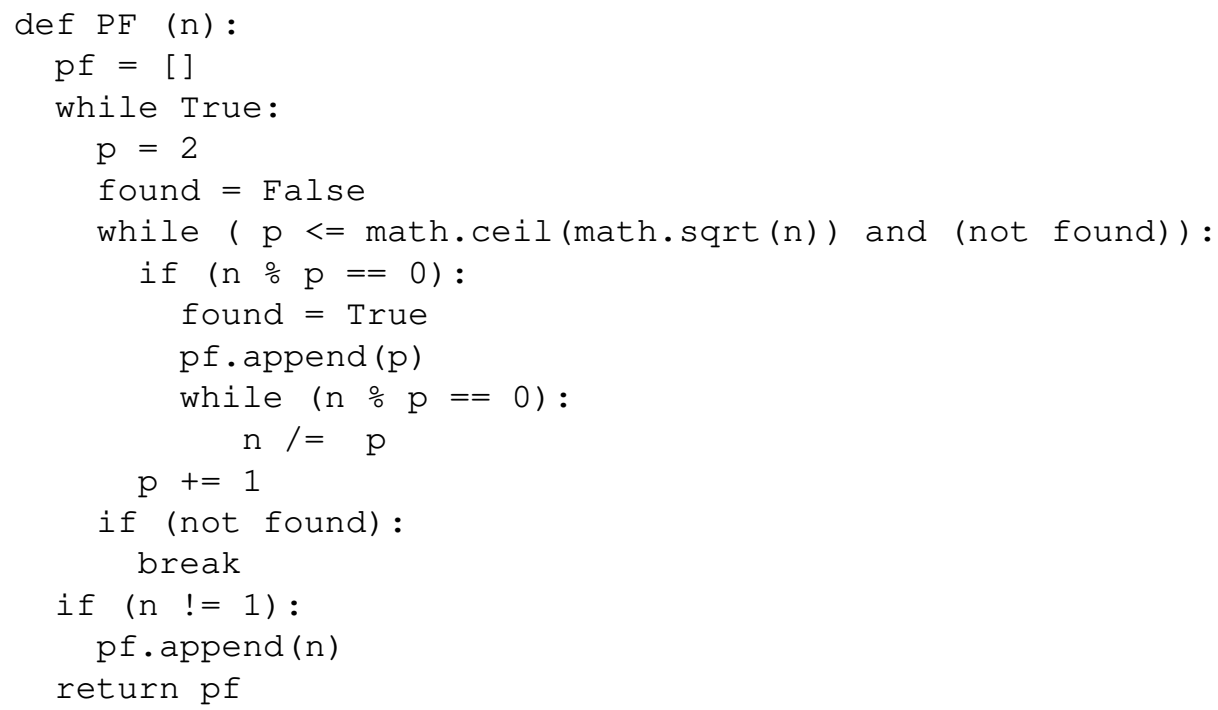

The method PF calculates the prime factors of $n$ by repeatedly finding the least prime $p$ dividing $n$ and factoring out the largest power of $p^{\alpha}$ which divides $n$. Then this process is repeated on $n / p^{\alpha}$.

As for the running time of PF, let $n=\prod_{i=1}^{k} p_{i}^{\alpha_{i}}$ be the prime factorization of $n$. The outer while loop executes $\omega(n)=k$ times, once for each $p_{i}$ dividing $n$, while one execution of the inner two while loops takes $O\left(\sqrt{n}+\alpha_{i}\right)$ time. Thus, the total run-time is $O\left(\sum_{p_{i} \mid n} \sqrt{n}+\alpha_{i}\right)=O\left(\sqrt{n} \omega(n)+\omega^{\prime}(n)\right)$. As $\omega(n) \in O(\log n / \log \log n)$ [3, Thm. 8.8.10] and $\omega^{\prime}(n) \in O(\log n)$ [12, Sect. 22.10], this gives the claimed worst case running time for PF.

Thus, the running time of is AbelPrim is $O(n \omega(n))$. Using the same estimate on the worst-case growth of $\omega(n)$, we obtain the following result:

Theorem 2. Given $x$, there is an algorithm to determine if $x \in A Q$ which runs in time $O\left(n \frac{\log n}{\log \log n}\right)$ time in the worst case.

For space complexity, we briefly note that the set $A Q$ is in DSPACE $(\log (n))$. To see this, if we are testing whether a word is of the form $u_{1} u_{2} \cdots u_{n}$ where $\Psi\left(u_{i}\right)=\Psi\left(u_{j}\right)$ for all $i, j$, we can use log-sized counters to keep track of the current prefix length $\left|u_{1}\right|$, block number $j(2 \leq j \leq n)$ and the values of the Parikh vectors for $u_{1}$ and $u_{j}$. Viewing the alphabet size as constant, this is a constant number of counters.

\subsection{A linear time algorithm for recognizing $A Q$}

We can improve the algorithm isAbelPrim from the previous section by caching commonly used Parikh vectors, and obtain a linear time algorithm. Let $\operatorname{gpf}(n)$ be the greatest prime factor of $n$. Then we note that if $\operatorname{gpf}(n)^{2} \mid n$, every $d \in D$ is divisible by $\operatorname{gpf}(n)$, while if $\operatorname{gpf}(n)^{2} \nmid n$, then every $d \in D$ is divisible by $\operatorname{gpf}(n)$ except $d=n / \operatorname{gpf}(n)$. In both cases, we will precompute the Parikh vectors of length $\operatorname{gpf}(n)$ in order to compute the Parikh vectors of length $d$ for all $d \in D$ which are divisible by $\operatorname{gpf}(n)$. 
Let $w$ be our input word of length $n$ and write $w=w_{1} w_{2} \cdots w_{n / \operatorname{gpf}(n)}$ where each block has length $\operatorname{gpf}(n)$. Let $\mathbf{u}_{i}=\Psi\left(w_{i}\right)$ for $1 \leq i \leq n / \operatorname{gpf}(n)$. Note then that if $\operatorname{gpf}(n) \mid d$, then the blocks of $w$ of length $d$ have Parikh vectors of the form

$$
\sum_{j=1}^{d / \operatorname{gpf}(n)} \mathbf{u}_{k d / \operatorname{gpf}(n)+j}
$$

for some $1 \leq k<\operatorname{gpf}(n)$. Thus, we can compute these Parikh vectors quickly by summing the precomputed $\mathbf{u}_{i}$.

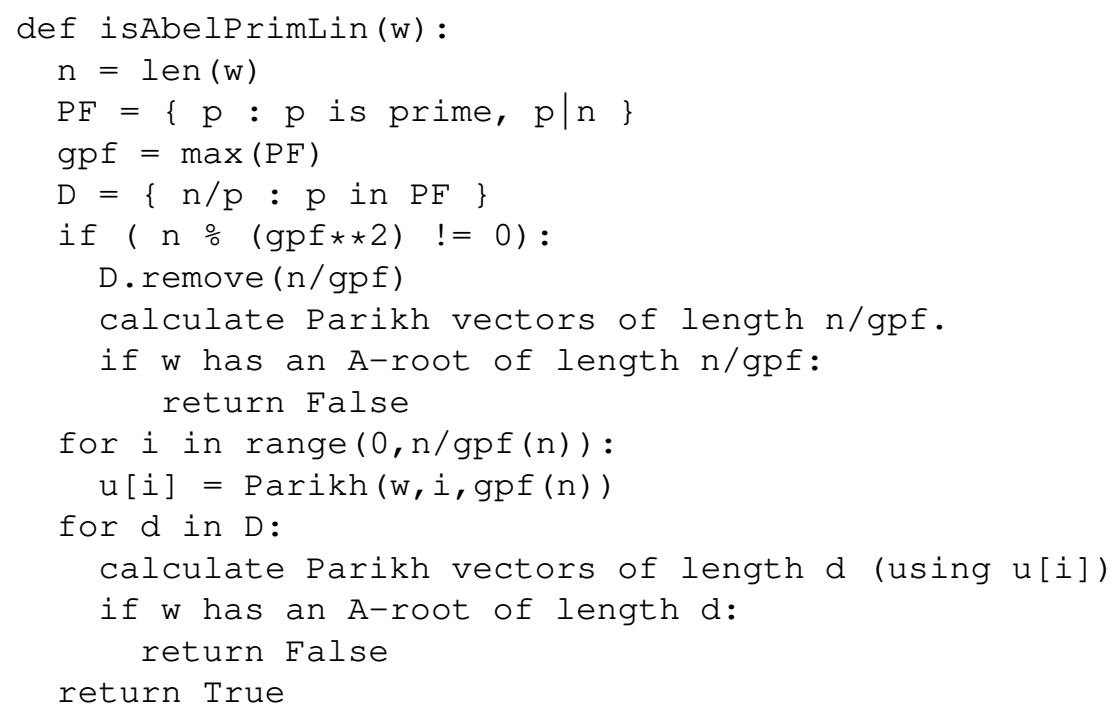

Here, we let Parikh $(w, i, j)$ be a method which computes the $i$-th Parikh vector of length $j$ in the word $w$.

This modified implementation has the same correctness as the previous implementation, as the same tests are performed. We now show the claimed $O(n)$ run time. Computing D and PF is the same as in is AbelPrim and can be done in linear time. Having computed PF, the calculation of $\operatorname{gpf}(n)$ takes $O(\omega(n))=O(\log n / \log \log n)$ time. In the case where $\operatorname{gpf}(n)^{2} \nmid n$, the time to execute the additional statements is $O(n)$ time. Similarly, the computation of the Parikh vectors u [i] takes time $O(n)$.

Consider the execution of the final for loop. For $d \in D$, we need $O(d / \operatorname{gpf}(n))$ time to compute one Parikh vector of a subword of $w$ of length $d$, so to compute all $n / d$ such vectors requires time $O(n / \operatorname{gpf}(n))$. To test the equalities of all these $n / d$ vectors (implied by the if statement) requires time $O(n / d)=O(p)$ where $d=n / p$. Thus, the worst case running time of the loop is

$$
\sum_{p \mid n}\left(O\left(\frac{n}{\operatorname{gpf}(n)}\right)+O(p)\right)=O\left(n \frac{\omega(n)}{\operatorname{gpf}(n)}\right)+O\left(\sum_{p \mid n} p\right) .
$$

We now estimate the first quantity.

Proposition 3. For all integers $n, \omega(n) / g p f(n) \leq 2 / 3$.

Proof. Note that if $\omega(n)=r$ for some integer $r$, then $\operatorname{gpf}(n) \geq p_{r}$ (where $p_{r}$ is the $r$-th prime), since if $n$ has $r$ prime factors, the minimum possible value for its largest prime factor is $p_{r}$. A simple induction proves that $p_{r}>2 r-1$ for $r \geq 2$. Thus, $\omega(n) / \operatorname{gpf}(n)$ is maximized by $x /(2 x-1)$ for all $n$ with at least two prime factors. But $\frac{x}{2 x-1}$ is maximized at $n=2$ on the interval $n \geq 2$. Thus, $\omega(n) / \operatorname{gpf}(n) \leq 2 / 3$ for all $n$ with at most two prime factors. For prime powers, $\omega(n) / \operatorname{gpf}(n) \leq 1 / n<2 / 3$.

Finally, we have that $\sum_{p \mid n} p \leq n$. Thus, the total running time of the loop is $O(n)$.

Theorem 3. Given $x$, there is an algorithm to determine if $x \in A Q$ which runs in time $O(n)$ time in the worst case. 


\section{Words with multiple A-primitive roots}

We show that unlike classical primitive words, a word may have multiple distinct A-primitive roots. This fact was essentially noted by Constantinescu and Ilie [5] who constructed an infinite word $w$ with two distinct Abelian periods. We generalize this to show that for all $n \geq 2$, we can construct a word with $n$ distinct A-primitive roots.

For all $n \geq 1$, let $Q_{n}=2 \cdot \prod_{i=1}^{n} p_{i}$, where $p_{i}$ is the $i$-th prime for $i \geq 1$, with $p_{1}=2$. Then for all $n \geq 1$, let $w_{n}$ be the word defined by

$$
w_{n}=a a b b(a b)^{\left(Q_{n}-4\right) / 2} .
$$

Note that $\left|w_{n}\right|=Q_{n}$. For example,

$$
w_{2}=a a b b a b a b a b a b .
$$

Lemma 1. For all $n \geq 2$, the word $w_{n}$ has $n$ distinct $A$-primitive roots. In particular, the words

$$
r_{m}=a a b b(a b)^{p_{m}-2}
$$

for all $n \geq m \geq 1$ are A-primitive roots of $w_{n}$.

Proof. First, note that $a b$ is not an A-primitive root of $w_{n}$, as it is not a prefix of $w_{n}$.

Let $1 \leq m \leq n$. Then the first subword of $w_{n}$ of length $2 p_{m}$ is $r_{m}$. All subsequent subwords of $w_{n}$ of length $2 p_{m}$ are $(a b)^{p_{m}}$. All subwords have Parikh vector $\left(p_{m}, p_{m}\right)$.

Finally, note that the lengths of $r_{m}$ form a division-free set $\left\{2 p_{n}: 2 \leq m \leq n\right\}$. Thus, all $r_{m}$ are A-primitive roots of $w_{n}$.

The following lemma shows that a word may not have A-primitive roots whose lengths are coprime.

Lemma 2. If $w$ has two distinct A-primitive roots $u, v$ where $|u|=\ell_{1},|v|=\ell_{2}$, then $\operatorname{gcd}\left(\ell_{1}, \ell_{2}\right) \geq 2$.

Proof. Assume that $w$ has two distinct A-primitive roots as above: $w=u_{1} u_{2} \cdots u_{m}$ and $w=v_{1} v_{2} \cdots v_{n}$ where $\left|u_{i}\right|=\ell_{1}$, $\left|v_{j}\right|=\ell_{2}$. Assume, contrary to what we want to prove that $\operatorname{gcd}\left(\ell_{1}, \ell_{2}\right)=1$.

First note that $m \geq \ell_{2}$. To see this, note that $|w|=m \ell_{1}=n \ell_{2}$ and we have that $\ell_{2} \mid \ell_{1} m$. If $m<\ell_{2}$, and as $\ell_{1}$ and $\ell_{2}$ are coprime, $\ell_{2} \mid \ell_{1} m$ is a contradiction.

Thus $m \geq \ell_{2}$ and $n \geq \ell_{1}$ as well. As $\operatorname{gcd}\left(\ell_{1}, \ell_{2}\right)=1$, there exist $r, s \geq 0$ such that $r \ell_{1}=s \ell_{2}-1$ (or $r \ell_{1}=s \ell_{2}+1$, which is proven similarly). As $m \geq \ell_{2}$ and $n \geq \ell_{1}$, we can assume that $s \leq n$ and $r \leq m$.

Thus, the prefix $v^{\prime}=v_{1} v_{2} \cdots v_{s}$ of $w$ of length $s \ell_{2}$ is one letter longer than the prefix $u^{\prime}=u_{1} u_{2} \cdots u_{r}$. Without loss of generality, let $a$ be the last symbol of $v_{s}$, which is also the first symbol of $u_{r+1}$. Let $\alpha=\left|u_{1}\right|_{a}$ and $\beta=\left|v_{1}\right|_{a}$. Counting the occurrences of $a$ in $u^{\prime}$ and $v^{\prime}$, we get

$$
r \alpha=s \beta-1 .
$$

Now consider that the prefix of $w$ of length $\ell_{1} \ell_{2}$ is $u_{1} \cdots u_{\ell_{2}}=v_{1} \cdots v_{\ell_{1}}$. Considering $v^{\prime \prime}=v_{s+1} \cdots v_{\ell_{1}}$ and $u^{\prime \prime}=$ $u_{r+1} \cdots u_{\ell_{2}}$, and again counting the occurrences of $a$, we also have

$$
\left(\ell_{2}-r\right) \alpha=\left(\ell_{1}-s\right) \beta+1 .
$$

Equating both (1) and (2) in terms of $\alpha$, we get

$$
r\left(\left(\ell_{1}-s\right) \beta+1\right)=\left(\ell_{2}-r\right)(s \beta-1) .
$$

Solving for $\beta$ gives $\beta=\ell_{2}$. Thus, we have that $v_{1} \in a^{+}$and thus $w$ only has $A$-primitive root $a$, a contradiction. Thus, $\operatorname{gcd}\left(\ell_{1}, \ell_{2}\right) \geq 2$.

\section{Number of Abelian Primitive Roots}

We now turn to the number of A-primitive roots a word may have, as a function of its length. As shown in the previous section, for any $n$, we can construct a word with $n$ A-primitive roots. In this section, we improve this to give a tight bound on the number of A-primitive roots a word may have. 


\subsection{Upper Bound}

We first give an upper bound on the number of A-primitive roots a word may have. We need an estimate $d(n)$ [3].

Theorem 4. The function $d(n)$ satisfies $d(n) \in O\left(2^{\log n / \log \log n}\right)$.

We will also use a result by de Bruijn et al. [8] (see also Anderson [2]):

Theorem 5. Let $n=p_{1}^{\alpha_{1}} p_{2}^{\alpha_{2}} \cdots p_{k}^{\alpha_{k}}$ be the prime factorization of $n \geq 2$. Let $D(n)$ be the set of integers defined by

$$
D(n)=\left\{p_{1}^{\beta_{1}} p_{2}^{\beta_{2}} \cdots p_{k}^{\beta_{k}}: \forall i\left(\beta_{i} \leq \alpha_{i}\right) \text { and } \sum_{i=1}^{k} \beta_{i}=\left\lfloor\omega^{\prime}(n) / 2\right\rfloor\right\} .
$$

Then $D(n)$ is a maximal anti-chain in the divisor lattice of $n$.

In other words, $D(n)$ is the largest division-free set of divisors of $n$. Anderson [2] gives the following estimate on the size of $D(n)$, which we denote $s(n)$ :

Theorem 6. Let $n=p_{1}^{\alpha_{1}} p_{2}^{\alpha_{2}} \cdots p_{k}^{\alpha_{k}}$ be the prime factorization of $n \geq 2$. Let $A(n)=\frac{1}{3} \sum_{i=1}^{k} \alpha_{i}\left(\alpha_{i}+2\right)$. Then the maximal anti-chain in the divisor lattice of $n$ has size $s(n)=\Theta(d(n) / \sqrt{A(n)})$.

Now a word $w$ of length $n$ has at most $|D(n)|$ A-primitive roots: if $r$ is an A-primitive root, then $|r|$ divides $|w|$ and $|r|$ is not divisible by the length of any other A-primitive root. Thus, we can obtain the following result:

Theorem 7. If $w$ is a word of length $n$, the number of distinct A-primitive roots is $s(n) \in o\left(2^{\log n / \log \log n}\right)$.

Proof. By Theorem 5, if $w$ is a word of length $n$, then $w$ has at most $s(n)$ distinct A-primitive roots. By Theorem 4 and Theorem $6, d(n) \in o\left(2^{\log n / \log \log (n)}\right)$. Thus, the result follows.

We can use a result of Anderson [1] which gives the average order of $s(n)$ :

Theorem 8. As $\omega^{\prime}(n) \rightarrow \infty$, we have

$$
s(n) \leq\left(\sqrt{\frac{2}{\pi}+o(1)}\right) \frac{d(n)}{\sqrt{\omega^{\prime}(n)}} .
$$

As $n \rightarrow \infty$,

$$
\sum_{m \leq n} \frac{d(m)}{\sqrt{\omega^{\prime}(m)}} \sim \frac{n \log n}{\sqrt{2 \log \log n}}
$$

\subsection{Lower Bound}

For a lower bound on the number of A-primitive roots a word may have, we give an explicit construction. For any $n \geq 2$, let $T(n)=\{k d: k \in \mathbb{N}, d \in D(n), k d \leq n\}$. Let $t_{1}<t_{2}<\cdots<t_{m_{n}}=n$ be the $m_{n}$ elements of $T(n)$ in sorted order. Define

$$
z_{n}=a^{t_{1}} b^{t_{1}} \prod_{i=2}^{m_{n}} a^{t_{i}-t_{i-1}} b^{t_{i}-t_{i-1}} .
$$

Note that $z_{n}$ is a word of length $2 n$ with $\Psi\left(z_{n}\right)=(n, n)$.

Example 2. If $n=30$, then $D(30)=\{2,3,5\}$. In this case

$$
T(30)=\{2,3,4,5,6,8,9,10,12,14,15,16,18,20,21,22,24,25,26,27,28,30\} .
$$

With this, we have

$$
z_{n}=\text { aabbababababaabbababaabbaabbababaabbaabbababaabbababababaabb. }
$$

Lemma 3. Let $n \geq 2$ and $t \in D(n)$. Then $z_{n}$ has an A-primitive root of length $2 t$. 
Proof. Let $1 \leq j \leq m_{n}$ be the index such that $t=t_{j}$. As $t \in D(n) \subset T(n)$, we have that the prefix of $z_{n}$ of length $2 t$ is

$$
w_{n}=a^{t_{1}} b^{t_{1}} a^{t_{2}-t_{1}} b^{t_{2}-t_{1}} \cdots a^{t-t_{j-1}} b^{t-t_{j-1}} .
$$

Note that $\Psi\left(w_{n}\right)=(t, t)$. Now, each additional block of length $2 t$ from has the form

$$
a^{t_{\alpha}} b^{t_{\alpha}} \cdots a^{t_{\beta}} b^{t_{\beta}}
$$

for some $\alpha, \beta$ which are differences of successive $t_{i}$. To see this, note that these factors of $z_{n}$ begin and end at positions which are multiples of $t \in D(n)$, so each of the breakpoints are elements of $T(n)$. By telescoping, each of these factors has Parikh vector $(t, t)$. Thus, $z_{n}$ is a $n / t$-th A-power.

Further, $w_{n}$ must be an A-primitive root of $z_{n}$. Otherwise, there is some $z \in T(n)$ such that $z \mid t$, but in this case, $z$ is divisible by some element in $D(n)$, by definition of $T(n)$. But this gives a contradiction, since $t \in D(n)$ and $D(n)$ is an anti-chain of divisors.

Corollary 1. For all $n \geq 2$, there exists a word of length $2 n$ with $s(n)$ distinct A-primitive roots.

\section{Counting Abelian Primitive Words}

Let $\psi_{k}(n)$ be the number of primitive words of length $n$ over a $k$-letter alphabet, $\psi_{k}^{A}(n)$ be the number of A-primitive words of length $n$ over a $k$-letter alphabet and $\Delta_{k}(n)=\psi_{k}(n)-\psi_{k}^{A}(n)$. Note that $\Delta_{k}(n) \geq 0$ for all $n$, but we can observe, e.g., that $\Delta_{k}(p)=0$ for all primes $p$. Small values of $\psi_{k}^{A}(n)$ are given in Figure 1

\begin{tabular}{|r|r|r|r|r|}
\hline$n k \rightarrow$ & 2 & 3 & 4 & 5 \\
\hline 1 & 2 & 3 & 4 & 5 \\
\hline 2 & 2 & 6 & 12 & 20 \\
\hline 3 & 6 & 24 & 60 & 120 \\
\hline 4 & 10 & 66 & 228 & 580 \\
\hline 5 & 30 & 240 & 1020 & 3120 \\
\hline 6 & 36 & 612 & 3792 & 15000 \\
\hline 7 & 126 & 2184 & 16380 & 78120 \\
\hline 8 & 186 & 5922 & 62820 & 382740 \\
\hline 9 & 456 & 19302 & 260952 & 1950420 \\
\hline 10 & 740 & 54300 & 1016880 & 9637400 \\
\hline 11 & 2046 & 177144 & 4194300 & 48828120 \\
\hline 12 & 2972 & 490488 & 16354320 & 241776440 \\
\hline 13 & 8190 & 1594320 & 67108860 & 1220703120 \\
\hline 14 & 12824 & 4509750 & 263483136 & \\
\hline 15 & 30030 & 14227920 & & \\
\hline 16 & 52666 & 40888962 & & \\
\hline 17 & 131070 & 129140160 & 17179869180 & 762939453120 \\
\hline 18 & 202392 & 368252856 & & \\
\hline 19 & 524286 & 1162261464 & 274877906940 & 19073486328120 \\
\hline 20 & 859180 & & & \\
\hline
\end{tabular}

Figure 1: Number of A-primitive words $\psi_{k}^{A}(n)$ by length $(n)$ and alphabet size $(k)$.

The function $\psi_{k}(n)$ is well-known (see, e.g., Lothaire [14]). The formula

$$
\psi_{k}(n)=\sum_{d \mid n} \mu(d) k^{n / d}
$$


expresses $\psi_{k}$ in terms of the Möbius function $\mu$ defined by $\mu(1)=1, \mu(n)=(-1)^{k}$ if $n$ is a product of $k$ distinct primes and $\mu(n)=0$ if $p^{2} \mid n$ for some prime $p$.

We can characterize $\Delta_{k}$ for prime powers exactly:

Lemma 4. For all primes $p$ and all $r \geq 2$,

$$
\Delta_{k}\left(p^{r}\right)=\sum_{n_{1}+n_{2}+\cdots+n_{k}=p^{r-1}}\left(\begin{array}{c}
p^{r-1} \\
n_{1} n_{2} \ldots n_{k}
\end{array}\right)\left(\left(\begin{array}{c}
p^{r-1} \\
n_{1} n_{2} \ldots n_{k}
\end{array}\right)^{p-1}-1\right) .
$$

Here, the sum is taken over all partitions $n_{1}+n_{2}+\cdots+n_{k}$ of $p^{r-1}$.

Proof. Let $x \in Q-A Q$ of length $p^{r}$. As $x$ is not A-primitive, it has a A-primitive root of length $p^{i}$ for some $1 \leq i<r$. But then $x$ can also be written as $x=x_{1} x_{2} \cdots x_{p}$ where $\left|x_{i}\right|=p^{r-1}$ and $\Psi\left(x_{i}\right)=\Psi\left(x_{j}\right)$ for all $1 \leq i, j \leq p$. Thus, it suffices to count only those $x$ of this form.

Consider that there are $\left(\begin{array}{c}p^{r-1} \\ n_{1} n_{2} \ldots n_{k}\end{array}\right)$ different words $x_{1}$ of length $p^{r-1}$ such that $\Psi\left(x_{1}\right)=\left(n_{1}, n_{2}, \ldots, n_{k}\right)$ for each partition $n_{1}+n_{2}+\cdots+n_{k}=n$. As recently noted by Richmond and Shallit [16], for a fixed choice of $x_{1}$, the remainder of the words $x_{2}, \ldots, x_{p}$ must satisfy $\Psi\left(x_{j}\right)=\Psi\left(x_{1}\right)$, which can be done in $\left(\begin{array}{c}p^{r-1} \\ n_{1} n_{2} \ldots n_{k}\end{array}\right)$ ways for each $2 \leq j \leq p$. Thus, we get a total of $\left(\begin{array}{c}p^{r-1} \\ n_{1} n_{2} \ldots n_{k}\end{array}\right)^{p-1}$ possibilities, and we must exclude the choice $x_{1}=x_{2}=x_{3}=\cdots=x_{p}$, as this word is not primitive.

Thus, multiplying the number of choices of the word $x_{1}$ and the words $x_{2}, \cdots, x_{p}$ and summing over all possible Parikh vectors, we get the result.

The problem of giving a closed form of $\Delta_{k}(n)$ or $\psi_{k}^{A}(n)$ for all values of $n$ is still open.

\section{Equivalence Relations on A-primitive words}

In this section, we consider classical results such as the Lyndon-Schützenberger Theorem for classical words in the context of Abelian primitivity. To do so, we define an appropriate equivalence relations to replace equality.

We first note that the A-primitive words are not closed under conjugation. For example, note that bbababaa $\in A Q$ but aabbabab $\notin A Q$. Because of this, the concept of a Lyndon-type word for A-primitive words is not a straight forward definition (recall that a primitive word $w$ is a Lyndon word if it is the lexicographically least word in its class of conjugates).

For all $n \geq 1$, let $\sim_{n}$ be the binary relation defined on words by $u \sim_{n} x$ if we can write $u=\alpha_{1} \alpha_{2} \cdots \alpha_{m}$ and $x=\beta_{1} \beta_{2} \cdots \beta_{m}$ where

(a) for all $1 \leq i \leq m,\left|\alpha_{i}\right|=\left|\beta_{i}\right|=n$.

(b) for all $1 \leq i, j \leq m, \Psi\left(\alpha_{i}\right)=\Psi\left(\beta_{j}\right)$. $\Psi$.

Thus, $\sim_{n}$ represents that two words can be broken into blocks of length $n$, all of which have the same image under

Example 3. Let $n=3$. Then $a b c a c b a b c \sim_{3}$ cbabcabca as each block $b$ of length three in both words satisfies $\Psi(b)=(1,1,1)$.

We use $\sim_{n}$ to investigate relationships with the theory of codes in the context of commutation.

Theorem 9. For all words $u, x \in \Sigma^{*}, u x \sim_{n} x u$ if and only if there exists $r \geq 1, \alpha_{1}, \ldots, \alpha_{r}, \beta_{1}, \ldots, \beta_{r} \in \Sigma^{*}$ such that

(a) for all $1 \leq i \leq r,\left|\alpha_{i} \beta_{i}\right|=n$.

(b) for all $1 \leq i, j \leq r, \Psi\left(\alpha_{i}\right)=\Psi\left(\alpha_{j}\right)$ and $\Psi\left(\beta_{i}\right)=\Psi\left(\beta_{j}\right)$.

(c) there exists $1 \leq s<r$ such that $u=\alpha_{1} \beta_{1} \cdots \alpha_{s-1} \beta_{s-1} \alpha_{s}$ and $x=\beta_{s} \alpha_{s+1} \beta_{s+1} \cdots \alpha_{r} \beta_{r}$. 
Proof. $(\Leftarrow)$ Let $u, x$ satisfy the conditions. Then we have that

$$
\begin{aligned}
u x & =\alpha_{1} \beta_{1} \cdots \alpha_{r} \beta_{r} \\
x u & =\left(\beta_{s} \alpha_{s+1}\right)\left(\beta_{s+1} \alpha_{s+2}\right) \cdots\left(\beta_{r-1} \alpha_{r}\right)\left(\beta_{r} \alpha_{1}\right)\left(\beta_{1} \alpha_{2}\right) \cdots\left(\beta_{s-2} \alpha_{s-1}\right)\left(\beta_{s-1} \alpha_{s}\right)
\end{aligned}
$$

Thus, note that with the parenthesization above, we have that each subword in $u x$ of length $n$ has the form $\alpha_{i} \beta_{i}$ subwords while in $x u$, they have the form $\beta_{i} \alpha_{i+1(\bmod r)}$.

Now note that for any value of $i$ and $j$, we have

$$
\Psi\left(\alpha_{i} \beta_{i}\right)=\Psi\left(\alpha_{i}\right)+\Psi\left(\beta_{i}\right)=\Psi\left(\alpha_{j+1(\bmod r)}\right)+\Psi\left(\beta_{j}\right)=\Psi\left(\beta_{j} \alpha_{j+1(\bmod r)}\right) .
$$

Thus, $u x \sim_{n} x u$.

$\Leftrightarrow$ Let $u x \sim_{n} x u$. Then we can write $u x=\gamma_{1} \gamma_{2} \cdots \gamma_{t}$ and $x u=\eta_{1} \eta_{2} \cdots \eta_{t}$ where for all $1 \leq i, j \leq t$, we have $\Psi\left(\gamma_{i}\right)=\Psi\left(\eta_{j}\right)$ and $\left|\gamma_{i}\right|=\left|\eta_{j}\right|=n$.

Assume without loss of generality that $|u|>|x|$. Let $1 \leq p<t$ be such that

$$
\begin{aligned}
u & =\gamma_{1} \gamma_{2} \cdots \gamma_{p} \gamma_{p+1}^{\prime} \\
x & =\gamma_{p+1}^{\prime \prime} \gamma_{p+2} \cdots \gamma_{t}
\end{aligned}
$$

where $\gamma_{p+1}^{\prime} \gamma_{p+1}^{\prime \prime}=\gamma_{p+1}$. Similarly, we can write

$$
\begin{aligned}
x & =\eta_{1} \eta_{2} \cdots \eta_{t-p-1} \eta_{t-p}^{\prime} \\
u & =\eta_{t-p}^{\prime \prime} \eta_{t-p+1} \cdots \eta_{t}
\end{aligned}
$$

where $\eta_{t-p}^{\prime} \eta_{t-p}^{\prime \prime}=\eta_{t-p}$

Thus, we have that $\gamma_{1} \gamma_{2} \cdots \gamma_{p} \gamma_{p+1}^{\prime}=\eta_{t-p}^{\prime \prime} \eta_{t-p+1} \cdots \eta_{t}$. Write $\eta_{t}=\eta_{t}^{\prime} \eta_{t}^{\prime \prime}$ where $\left|\eta_{t}^{\prime \prime}\right|=\left|\gamma_{p+1}^{\prime}\right|$. Similarly, write $\gamma_{p}=\gamma_{p}^{\prime} \gamma_{p}^{\prime \prime}$ where $\left|\gamma_{p}^{\prime \prime}\right|=\left|\eta_{t}^{\prime}\right|$. Then we have that $\eta_{t}=\gamma_{p}^{\prime \prime} \gamma_{p+1}^{\prime}$ and so certainly their images under $\Psi$ are the same. As all blocks of $u x$ and $x u$ have the same image, we can therefore conclude that $\Psi\left(\gamma_{p+1}\right)=\Psi\left(\gamma_{p}^{\prime \prime} \gamma_{p+1}^{\prime}\right)$. But clearly $\Psi\left(\gamma_{p+1}\right)=\Psi\left(\gamma_{p+1}^{\prime}\right)+\Psi\left(\gamma_{p+1}^{\prime \prime}\right)$. Therefore, we get that $\Psi\left(\gamma_{p+1}^{\prime \prime}\right)=\Psi\left(\gamma_{p}^{\prime \prime}\right)$. Finally, we have that $\Psi\left(\gamma_{p}\right)=\Psi\left(\gamma_{p+1}\right)$ gives that $\Psi\left(\gamma_{p}^{\prime}\right)=\Psi\left(\gamma_{p+1}^{\prime}\right)$. Continuing in this way, we can factorize each $\gamma_{i}$ into $\gamma_{i}^{\prime}$ and $\gamma_{i}^{\prime \prime}$ so that all $\gamma_{i}^{\prime}$ have the same image under $\Psi$, and separately, all the $\gamma_{i}^{\prime \prime}$ have the same image under $\Psi$.

Thus, let $t=r, s=p$ and $\alpha_{i}=\gamma_{i}^{\prime}$ and $\beta_{i}=\gamma_{i}^{\prime \prime}$ for all $1 \leq i \leq r$. Then we get that $u=\alpha_{1} \beta_{1} \cdots \alpha_{s} \beta_{s} \alpha_{s+1}$ and $x=\beta_{s} \alpha_{s+1} \beta_{s+1} \cdots \alpha_{r} \beta r$. We can then verify that the remaining conditions of the lemma hold using these definitions of $\alpha_{i}, \beta_{i}$.

Example 4. If $x=a b c a$ and $u=c b a b c$ then $x u \sim_{3} u x$ (which was shown in Example 3). Note that $x$ and $u$ have different lengths and thus cannot share an A-primitive root.

The case where both $x$ and $u$ have A-primitive roots of length $n$ is of particular interest:

Corollary 2. Let $u, x \in \Sigma^{*}$ with $u x \sim_{n} x u$. If $u$ has an A-primitive root of length $n$, then $x$ does as well, and these A-roots are the same.

Corollary2]is analogous to the second Lyndon-Schützenberger theorem (see e.g., Lothaire [14] or Shyr [18]) which can be interpreted (in part) as $u x=x u$ if and only if $x$ and $u$ both have the same primitive root.

We note that the conditions of $\sim_{n}$ cannot be weakened to allow not all of the subwords of both $u$ and $x$ to have identical images under $\Psi$ and have Theorem 9 holds, as the following example demonstrates:

Example 5. Let $\simeq_{n}$ be the binary relation defined on words by $u \simeq_{n} x$ if we can write $u=\alpha_{1} \alpha_{2} \cdots \alpha_{m}$ and $x=$ $\beta_{1} \beta_{2} \cdots \beta_{m}$ where

(a) for all $1 \leq i \leq m,\left|\alpha_{i}\right|=\left|\beta_{i}\right|=n$.

(b) for all $1 \leq i \leq m, \Psi\left(\alpha_{i}\right)=\Psi\left(\beta_{i}\right)$.

Thus, only parallel subwords of length $n$ are required to be permutations of one another in this definition. But note that if $x=a$ and $u=b a a$ then $a b a a \simeq_{2} b a a a$. Note that no factorization of $x$ and $u$ of the form of Theorem 9 can exist, as $u$ cannot be factored as $u=\beta u^{\prime} \beta$ for any nonempty word $\beta$. 


\section{Conclusions}

We have studied the formal language theoretic and combinatorial properties of Abelian primitive words. Unlike classical primitive words, the number of Abelian primitive words is a nontrivial combinatorial problem. On the other hand, we show that the set of Abelian primitive words are not context-free, unlike the long-standing open problem for primitive words. Future research problems include an exact enumeration of the number of Abelian primitive words of length $n$.

\section{References}

[1] I. Anderson. On primitive sequences. Journal London Math. Soc, 42:137-148, 1967.

[2] I. Anderson. Combinatorics of finite sets. Dover Publications, 2002.

[3] E. Bach and J. Shallit. Algorithmic Number Theory, volume 1. MIT Press, 1997.

[4] J. Berstel and L. Boasson. The set of Lyndon words is not context-free. Bull. EATCS, 63:139-140, 1997.

[5] S. Constantinescu and L. Ilie. Fine and Wilf's theorem for Abelian periods. Bull. EATCS, 89:167-170, 2006.

[6] R. Crandall and C. Pomerance. Prime Numbers: A Computational Perspective. Springer, 2nd edition, 2005.

[7] E. Czeizler, L. Kari, and S. Seki. On a special class of primitive words. Theoretical Computer Science, 411:617$630,2010$.

[8] N. de Bruijn, C. van Ebbenhorst Tengbergen, and D. Kruyswijk. On the set of divisors of a number. Nieuw Arch. Wiskunde, 23:191-193, 1951.

[9] M. Domaratzki. Trajectory-Based Operations. PhD thesis, Queen's University, 2004.

[10] P. Dömösi, S. Horváth, M. Ito, L Kászonyi, and M. Katsura. Formal languages consisting of primitive words. In Z. Ésik, editor, Fundamentals of Computation Theory, 9th International Symposium, FCT '93, Szeged, Hungary, August 23-27, 1993, Proceedings, volume 710 of Lecture Notes in Computer Science, pages 194-203. Springer, 1993.

[11] J. Gabarró. Some applications of the interchange lemma. Bull. EATCS, 25:19-21, 1985.

[12] G. Hardy and E. Wright. An Introduction to the Theory of Numbers. Oxford Science Publications, 5th edition, 2000.

[13] J. Jędrzejowicz and A. Szepietowski. On the expressive power of the shuffle operator matched with intersection by regular sets. Theoretical Informatics and Applications, 35:379-388, 2001.

[14] M. Lothaire. Combinatorics on words. Cambridge University Press, 1997.

[15] H. Petersen. The ambiguity of primitive words. In P. Enjalbert, E. Mayr, and K. Wagner, editors, STACS 94, 11th Annual Symposium on Theoretical Aspects of Computer Science, Caen, France, February 24-26, 1994, Proceedings, volume 775 of Lecture Notes in Computer Science, pages 679-690, 1994.

[16] L. B. Richmond and J. Shallit. Counting Abelian squares. Elec. J. Combinatorics, 16:R72, 2009.

[17] G. Rozenberg and A. Salomaa. Handbook of Formal Languages, volume 1. Springer, 1997.

[18] H. Shyr. Free Monoids and Languages. Hon Min Book Company, 3rd edition, 2001. 